\title{
p2pCM: A Structured Peer-to-Peer Grid Component Model
}

\author{
Carles Pairot ${ }^{1}$, Pedro García ${ }^{1}$, Rubén Mondéjar, and Antonio F. Gómez Skarmeta ${ }^{2}$ \\ ${ }^{1}$ Department of Computer Science and Mathematics, Universitat Rovira i Virgili \\ Avinguda dels Països Catalans 26, 43007 Tarragona, Spain \\ \{carles.pairot, pedro.garcia\}@urv.net \\ ${ }^{2}$ Department of Computer Engineering, Universidad de Murcia \\ Apartado 4021, 30001 Murcia, Spain \\ skarmeta@fcu.um.es
}

\begin{abstract}
In this paper we present $\mathrm{p} 2 \mathrm{pCM}$, a new distributed componentoriented model aimed to structured peer-to-peer grid environments. Our model offers innovative contributions like a lightweight distributed container model, an adaptive component activation mechanism, which takes into account network proximity, and a decentralized component location and deployment service. We believe that all of the features our component-oriented model provides can be very promising for the development of future wide-area distributed applications.
\end{abstract}

\section{Introduction}

Developers face an important problem when willing to build wide-area componentbased distributed applications. Practically there are no frameworks oriented to such target market. In this paper we present p2pCM: a peer-to-peer component model for building distributed component-based applications on top of a structured peer-topeer grid. Our model offers traditional and novel component services by means of an underlying object middleware (Dermi [3]). To the best of our knowledge, this is the first component model built on top of a structured P2P grid infrastructure.

The main contributions of this paper are the design of a new wide-area component model that runs on top of a structured P2P grid infrastructure; the utilization of a decentralized component location and deployment facility; and the mplementation of a resilient and autonomous lightweight container model, which provides component's life cycle services, and many others. Component instantiation is of special interest because it takes into account network locality properties.

The rest of the paper is structured as follows, in Section 2 we give an overview of p2pCM's architecture and services, in Section 3 we analyze possible usage scenarios for our component model, and in Section 4 we explore related work. We conclude in Section 5 by providing an outline of future work.

\section{2 p2pCM Architecture and Services}

The decentralized component model we have designed ( $\mathrm{p} 2 \mathrm{pCM}$ ) runs on top of a structured peer-to-peer grid. We have implemented the majority of traditional compo- 
nent models services, and adapted them to the underlying topology. These include a decentralized component location and deployment facility, and a decentralized lightweight container model, which provides a component persistence and life cycle service, as well as an adaptive activation policy.

\subsection{Decentralized Component Location and Deployment}

All components must be previously registered into the system so as they can be used by any client. This deployment phase is found in all traditional component models, as well as in ours. In our model, we use a decentralized naming service which stores all component's metadata (read in deployment time), as well as the component's class files. This is done to allow dynamic component class loading in clients that do not have the necessary component classes. Our naming service benefits from the efficient overlay network routing properties thus hashing the component's identifier, and storing the values into the node whose key is closer to this hash. Fault tolerance is provided by he underlying Dermi [3] middleware in a transparent way to the developer.

\subsection{Decentralized Container Model}

In $\mathrm{p} 2 \mathrm{pCM}$ we have tried to avoid heavy and monolithic containers used by traditional component models (e.g. J2EE or CORBA), and opted for designing a decentralized lightweight container model. In our case, all of the nodes that belong to the network are containers, and as such, they can house components. The idea is that any component can be run in any node (unless restricted by security constraints), because each node runs a lightweight container. Our containers are fault resilient and autonomous: components are replicated all along the network using the underlying Dermi [3] middleware. If a container fails, surely other containers housing those components will exist in the network. Now we are going to briefly describe the different services our decentralized container model offers to components.

\subsubsection{Component Life Cycle Service}

The container is responsible for activation and passivation of components. When creating a component's instance, the following may happen:

- No other component instance is already active in the network. In this case, the instance will be activated on our local container, and a local reference is returned so as we can interact with it.

- Other component instances are already active in the network. In such case, we will get a reference to the closest instance (basically, a Dermi anycall is done). However, if this closest instance informs us that it cannot accept more requests (it may be overwhelmed), a local copy is activated.

Component instances are passivated when they are not called during a certain amount of time in order to save resources on the node.

\subsubsection{Component Persistence}

Stateful components need to be ensured state persistence. p2pCM allows this by providing a persistence mechanism in order to take care of total passivation of component instances. If all component instances are passivated, their shared state is lost, and 
this situation is not desired. The persistence strategy is chosen by the component itself by means of the persistence metadata tag in the deployment phase. Persistence itself is managed by the decentralized object replication mechanisms of Dermi.

\subsubsection{Adaptive Component Activation}

In wide-area environments, some component instances could become temporarily overwhelmed with requests of nearby clients. To avoid such problem, we use an adaptive component activation mechanism.

The idea is to know which immediate node is delivering most messages to the overwhelmed node (via the getPreviousHopHandle() method), and start an algorithm which keeps activating new component instances all along the overwhelming request path, until stress is relieved.

We are currently working in this adaptive activation scheme in order to fine tune several parameters, and as future work we will perform extensive simulations to validate our approach.

\section{3 p2pCM Usage Scenario}

One scenario where our model could be successfully applied would be in an application similar to a SETI@Home or United Devices Cancer Research Project model. Such applications normally require a central server which distributes computing units to home computers for analyzing. Our component model could be used to build an application which would efficiently be fault tolerant and resilient to high request peaks.

The idea would be that interested nodes would activate a Processing component on their local containers. This component could thus be receptive to a DataFeed component's calls. The Processing components would be aligned into a multicast group, so as the data feeder requested data unit analysis by anycalling or multicalling [3] to the Processing component group.

Once each Processing component finished its data unit analysis, instead of returning data back to the central DataFeed component (which would possibly create an important bottleneck there), it would pass data to another Results component by means of an event. This component would manage the results persistence by replicating itself and storing state information in the $\mathrm{P} 2 \mathrm{P}$ grid infrastructure. Whenever the DataFeed component wished to obtain the results (cyclically every $x$ hours), it would anycall to its closest Result component instance, which would provide it with the latest gathered results.

\section{Related Work}

In the Grid world there exist solutions like Fractal/ProActive [1], which is a hierarchical and dynamic component model. Nevertheless, its approach is different form $\mathrm{p} 2 \mathrm{pCM}$, in the sense that virtual node and virtual machine mapping is performed on the component's deployment descriptor, thus not allowing self adaptation to node failures. 
P2PComp [2] is built on top of an unstructured P2P network, and it is mainly aimed to address the development needs for mobile P2P applications. It features a lightweight container model as well, and provides many services, including synchronous / asynchronous remote invocations, hot swapping, service fetching and ranking. All these services are basically oriented for highly mobile and dynamic applications.

\section{Conclusions and Future Work}

In this paper we have presented $\mathrm{p} 2 \mathrm{pCM}$, a decentralized component model built on top of a structured P2P Grid. Our approach manages to provide many interesting services to component developers. Our main contributions include a decentralized component location and deployment facility, and the adoption of a decentralized lightweight and fault resilient container model. We have shown as well, possible usage scenarios of our system. However, we still do not provide other services found in commercial component-oriented models, like security or transactions, which need to be further investigated.

A first prototype of $\mathrm{p} 2 \mathrm{pCM}$ is available through http://ants.etse.urv.es/p2pcm. We believe that the features $\mathrm{p} 2 \mathrm{pCM}$ offers can be very promising for the development of future wide-area distributed applications.

This work has been partially funded by the Spanish Ministry of Science and Technology through project TIC-2003-09288-C02-00.

\section{References}

1. Baude, F., Caromel, D., and Morel, M., "From Distributed Objects to Hierarchical Grid Components", Proc. DOA 2003, LNCS, pp. 1226-1242, Nov. 2003.

2. Ferscha, A., Hechinger, M., et al, "A Light-Weight Component Model for Peer-to-Peer Applications”, Proc. of ICDCS 2004, pp. 520-527.

3. Pairot, C., García, P., and Gómez Skarmeta, A. F., "Dermi: A New Distributed Hash Tablebased Middleware Framework”. IEEE Internet Computing. Vol 8, No. 3, 2004. 